\title{
Ferromagnetic EuS films: Magnetic stability, electronic structure and magnetic surface states
}

\author{
Wolf Müller* and Wolfgang Nolting \\ Lehrstuhl Festkörpertheorie, Institut für Physik, Humboldt-Universität zu Berlin, Newtonstraße 15, 12489 Berlin, Germany
}

\begin{abstract}
We present the temperature and layer dependent electronic structure of a 20-layer EuS(100)-film using a combination of first-principles and model calculation, the latter based on the ferromagnetic Kondo-lattice. The calculated thickness-dependent Curie temperature agrees very well with experimental data. The projected 5d-bandstructure is at finite temperatures strongly influenced by damping effects due to spin exchange processes. Spin-split unoccupied 5d-surfaces states are found with a Stoner-like collapsing for increasing temperature towards the Curie point and with an exponential decay of spectral weight with increasing distance from the surface.
\end{abstract}

PACS numbers: 71.27.+a,73.20.At,75.50.Pp,75.70.Ak

Europiumsulphide is a prototype ferromagnetic semiconductor, crystallizing in the rocksalt structure with a lattice constant $a=5.95 \AA$. The $\mathrm{Eu}^{2+}$ ions occupy lattice sites of an fcc structure. The just half-filled $4 \mathrm{f}$ shell of the rare earth ion creates a strictly localized magnetic moment of $7 \mu_{B}$ according to the ground state configuration ${ }^{8} S_{7 / 2}$. The moments are exchange coupled resulting in a ferromagnetic order for temperatures below the Curie temperature $T_{\mathrm{C}}=16.57 \mathrm{~K}[1]$. As to the purely magnetic properties bulk-EuS is considered rather well understood being an almost ideal realization of the Heisenberg model, where the exchange integrals can be restricted to nearest $\left(J_{1} / k_{B}=0.221 \mathrm{~K}\right)$ and next nearest neighbors $\left(J_{2} / k_{B}=-0.100 \mathrm{~K}\right)$, only [2].

Recently, there has been an increasing interest in thin EuS films, in particular what concerns the thickness dependence of the ferromagnetic transition temperature [3] which is frequently discussed in terms of finite-size scaling (dimensionality crossover) [4]. Investigations of this kind have already earlier been done for films of the metallic counterpart Gadolinium [5]. In addition, Gd has provoked numerous research activities with respect to its extraordinary magnetic surface properties as, e. g., a possibly enhanced Curie temperature of the (0001) surface compared to that of bulk Gd [6].

While the Heisenberg model provides an excellent description of the purely magnetic properties of localmoment insulators such as EuS, $\mathrm{EuO}$ or metals like Gd it is of course inadequate for describing electronic and magnetooptic effects. So it cannot explain the striking temperature dependence of the $5 \mathrm{~d}$ conduction bands, first observed for the ferromagnetic europium compounds as red shift of the optical absorption edge for electronic $4 \mathrm{f}-$ $5 \mathrm{~d}$ transitions upon cooling below $T_{\mathrm{C}}[1]$. The reason is an interband exchange coupling of the excited 5d electron to the localized $4 \mathrm{f}$ moments that transfers the temperature dependence of the ferromagnetic moment state to the unoccupied conduction band states. Recently the same temperature effects have been verified for film structures in the case of $\mathrm{EuO}$ by spin-resolved $\mathrm{x}$-ray absorption spectroscopy 7] and by quasiparticle band structure calculations [8, 9]. In ref. 9] the theoretical prediction of a magnetic surface state has led to the speculation that the temperature dependence below $T_{\mathrm{C}}$ may give rise to a surface insulator-halfmetal transition accompanied by a huge magnetoresistance effect. It is one of the goals of our present study to teach whether this spectacular proposal may hold for EuS too.

We present in this paper a study of the temperature and layer dependent electronic structure of thin EuSfilms by combining a many-body model with a firstprinciples bandstructure calculation. The final goal are statements about magnetic stability in terms of the Curie temperature and the existence of surface states.

In the following we assume EuS film structures with two surfaces parallel to the fcc(100) crystal plane and consisting altogether of d equivalent parallel layers. The lattice sites $\mathbf{R}_{i \alpha}$ within the film are indicated by letters $\alpha, \beta, \ldots$ denoting the layer, and by letters $\mathrm{i}, \mathrm{j}, \ldots$ numbering the sites within a given layer, for which we assume translational symmetry in two dimensions. Each lattice site is occupied by a magnetic moment, represented by a spin operator $\mathbf{S}_{i \alpha}$, due to the half-filled $4 \mathrm{f}$ shell of the $\mathrm{Eu}^{2+}$ ion. The exchange coupled moments (spins) are certainly well described by a Heisenberg Hamiltonian:

$$
H_{4 \mathrm{f}}=\sum_{i j \alpha \beta} J_{i j}^{\alpha \beta} \mathbf{S}_{i \alpha} \mathbf{S}_{j \beta}-D_{0} \sum_{i \alpha}\left(S_{i \alpha}^{z}\right)^{2}
$$

As mentioned the exchange integrals $J_{i j}^{\alpha \beta}$ can be restricted to nearest $\left(J_{1}\right)$ and next nearest $\left(J_{2}\right)$ neighbors. The dipolar energy of EuS films is taken into account by a single-ion anisotropy $D_{0}$. This helps to overcome the Mermin-Wagner theorem [10] which forbids a collective moment order in films of finite thickness with isotropic Heisenberg exchange. Since the exchange constants are derived from a low-temperature spin wave analysis [2], we fix $D_{0}$, somewhat arbitrarily, by the requirement that our theory yields the experimental $T_{\mathrm{C}}$ for bulk EuS. We find $D_{0} / k_{B}=0.375 \mathrm{~K}[11$.

For the $5 \mathrm{~d}$ conduction electrons we use the partial op- 
erator $H_{5 d}$ :

$$
H_{5 \mathrm{~d}}=\sum_{i j \alpha \beta} \sum_{m m^{\prime}} T_{i j \alpha \beta}^{m m^{\prime}} c_{i \alpha m \sigma}^{\dagger} c_{j \beta m^{\prime} \sigma}
$$

$c_{i \alpha m \sigma}^{\dagger}\left(c_{i \alpha m \sigma}\right)$ is the creation (annihilation) operator of an electron with spin $\sigma$ at site $\mathbf{R}_{i \alpha}$ in the orbital $\mathrm{m}$. The $T_{i j \alpha \beta}^{m m^{\prime}}$ describe the electron hopping from $\mathbf{R}_{j \beta}$ to $\mathbf{R}_{i \alpha}$ with a possible orbital change $\left(m^{\prime} \rightarrow m\right)$. We require that these single-electron energies do not only account for the kinetic energy and the influence of the lattice potential, but also for all those interactions which are not explicitly covered by the model Hamiltonian. Therefore we take them from an LDA calculation. Details are given below.

Many important properties of local-moment materials such as EuS can be traced back to the interaction between the $4 \mathrm{f}$ and $5 \mathrm{~d}$ partial systems. Starting from the very general on-site Coulomb interaction between electrons of different orbitals it can be shown [8, 12] for the special case of EuS (half-filled spin-polarized $4 \mathrm{f}$ shell, empty conduction bands) that the interband interaction can be written as:

$$
\begin{aligned}
H_{4 \mathrm{f}-5 \mathrm{~d}}=-\frac{1}{2} J \sum_{i m \alpha}\left(S_{i \alpha}^{z}\left(n_{i m \alpha \uparrow}-n_{i m \alpha \downarrow}\right)\right. \\
\left.+S_{i \alpha}^{+} c_{i m \alpha \downarrow}^{\dagger} c_{i m \alpha \uparrow}+S_{i \alpha}^{-} c_{i m \alpha \uparrow}^{\dagger} c_{i m \alpha \downarrow}\right)
\end{aligned}
$$

$\mathrm{J}$ is the corresponding exchange coupling constant. Furthermore, we have used the standard abbreviations: $n_{i m \alpha \sigma}=c_{i m \alpha \sigma}^{\dagger} c_{i m \alpha \sigma} ; S^{ \pm}=S^{x} \pm \mathrm{i} S^{y}$. The first term in (31) describes an Ising-like interaction between the localized $4 \mathrm{f}$ spin and the spin of the itinerant $5 \mathrm{~d}$ electron, while the two others provide spin exchange processes between the two subsystems. Spin exchange may happen in three different elementary processes: magnon emission by an itinerant $\downarrow$ electron, magnon absorption by an $\uparrow$ electron and formation of a quasiparticle (magnetic polaron). The latter can be understood as a propagating electron dressed by a virtual cloud of repeatedly emitted and reabsorbed magnons corresponding to a polarization of the localized-spin neighborhood.

We believe that the total model Hamiltonian

$$
H=H_{4 \mathrm{f}}+H_{5 \mathrm{~d}}+H_{4 \mathrm{f}-5 \mathrm{~d}}
$$

incorporates the main physics of the ferromagnetic localmoment insulator EuS. It can be considered as the multiband version of the ferromagnetic Kondo-lattice model (KLM). To get a realistic description of EuS we try to cover all those interactions, which do not explicitly appear in our model Hamiltonian, by a proper renormalization of the single-particle energies. For this reason we performed a band-structures calculation using the tightbinding LMTO-atomic sphere approximation program of Andersen [13, 14]. Difficulties typical of LDA arise with the localized character of the $4 \mathrm{f}$ levels. A "normal" LDA calculation produces wrong $4 \mathrm{f}$ positions. To circumvent the problem we consider all the seven $4 \mathrm{f}$ electrons as core electrons in one spin channel, since the $4 \mathrm{f}$ levels enter our study only as localized spins in the sense of $H_{4 \mathrm{f}}$ in (1). We have to choose the proper single-particle input in such a way, that a double counting of just the decisive interband exchange (3), explicitly by the model Hamiltonian (44) and implicitly by the LMTO input, is avoided. The most direct solution of this problem would be to switch off the interband exchange $H_{4 \mathrm{f}-5 \mathrm{~d}}$ in the LDA code, what turns out to be impossible. We can exploit the fact that the non-trivial many-body problem of the KLM is exactly solvable for a ferromagnetically saturated semiconductor, e. g. EuS at $T=0 \mathrm{~K}[15]$. The result is especially simple for the $\uparrow$ spectrum because the $\uparrow$ electron cannot exchange its spin with the fully aligned $4 \mathrm{f}$ spins. Only the Ising term in $H_{4 \mathrm{f}-5 \mathrm{~d}}$ takes care for a rigid shift of the total $\uparrow$ spectrum by $\frac{1}{2} J S$. Thus we can use without any manipulation the $\uparrow$ dispersions of the LMTO calculation, which by definition works for $T=0 \mathrm{~K}$, for the EuS-hopping integrals in (2). There is no need to switch off $H_{4 \mathrm{f}-5 \mathrm{~d}}$ because in this special case it leads only to an unimportant rigid shift. It should be stressed, however, that the $\downarrow$ spectrum, on the contrary, is strongly influenced by the interband exchange, even at $T=0 \mathrm{~K}$ where it is still exactly calculable [15] (see fig. 4).

Because of the absence of conduction electrons in the semiconductor EuS, at least at low temperatures, the magnetic ordering of the localized $4 \mathrm{f}$ moments will be unaffected by the band states, because an RKKY-type contribution cannot occur. On the other hand the relevant superexchange mechanism is hidden in the exchange integrals $J_{i j}^{\alpha \beta}$ in (1), which are taken from the experiment 2] The magnetic part of the problem can therefore be solved separately via the extended Heisenberg Hamiltonian $H_{4 f}$. We employ a generalized version of the well-known Tyablikow approach, from which we know that it yields convincing results in the low- as well as high-temperature region. Details of the method are presented in [12]. The result is a distinctly layer- and temperature-dependent magnetization of the $4 \mathrm{f}$ moment system. The Curie temperature of the EuS-film exhibits a strong thickness-dependence, starting at about $2 \mathrm{~K}$ for the monolayer and approaching the bulk-value for more than 25 monolayers (Fig. 1). The $T_{\mathrm{C}}$ of the 20 layer film, we are going to discuss below, amounts to $16.28 \mathrm{~K}$. We find a remarkable agreement of our theoretical results with experimental data found by Stachow-Wojcik et al. 3]. A similar $T_{\mathrm{C}}$ behavior, derived from susceptibility measurements, is reported by Farle et al. [5] for the metallic counterpart Gd. The decrease of $T_{\mathrm{C}}$ with decreasing film thickness is understood as typical finite size scaling accompanied by a dimensionality cross-over.

To obtain the temperature-dependent electronic structure of a $\operatorname{EuS}(100)$ film the $T=0-\uparrow$ dispersions from our tight binding-LMTO band calculation are needed as single-particle input for the model evaluation. To ac- 


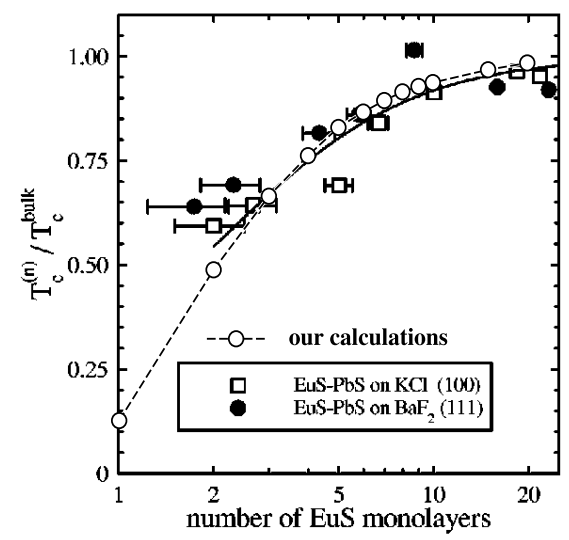

FIG. 1: Curie temperature as function of film thickness (number of EuS monolayers). Our theoretical results: $\circ-{ }_{-} \circ$. The experimental data are taken from ref. [3]. $T_{\mathrm{C}}^{\mathrm{bulk}}=16.57 \mathrm{~K}$.

count for the film geometry, we define a supercell consisting of $\mathrm{n}$ consecutive $\operatorname{EuS}(100)$ layers followed by $\mathrm{m}$ layers of empty spheres, i. e. periodically stacked EuS nlayer films, isolated from each other by m layers of empty spheres. It turned out that $m=5$ is large enough to guarantee truly isolated EuS films. We do not consider surface relaxation and reconstruction, which might be present in EuS films.

Similarly to the magnetic part the electronic part can be treated separately because magnon energies are smaller by some orders of magnitude compared to other energy terms as the exchange coupling constant $J$ or the conduction band width. So we can disregard the moment operator $H_{4 \mathrm{f}}$ when calculating the electronic self energy. That does not at all mean that the magnetic 4f moments do not influence the electronic quasiparticle spectrum. That is rather done by local-spin correlations such as $\left\langle S^{z}\right\rangle,\left\langle S^{ \pm} S^{\mp}\right\rangle,\left\langle\left(S^{z}\right)^{2}\right\rangle, \cdots$, which are to a great extent responsible for the temperature-dependence of the electronic spectrum and have to be derived from $H_{4 \mathrm{f}}$. To get the electronic self energy we apply the momentconserving decoupling approximation (MCDA) for suitably defined Green functions [8, 16]. In the set of equations which constitute the formal solution for the electronic self energy there appear the just mentioned $4 \mathrm{f}-$ spin correlations. Although the MCDA is partly based on an intuitive ansatz, it has been proved to be quite a reliable approach to the sophisticated many-body problem of the multiband-KLM. Interpolating self energy approaches [17], which fulfill a maximum number of exact limiting cases, as well as a systematic projection operator method [18] yield essentially identical results. We do not present details of the MCDA but refer the reader to ref. [8, 16].

In summary our theory contains only one parameter, namely the exchange coupling $J$. We assume that it is the same value for the film as for bulk-EuS, $J=0.23 \mathrm{eV}$ 11. Note that $J$ is not really a free parameter but being read off from the LDA band structure calculation.

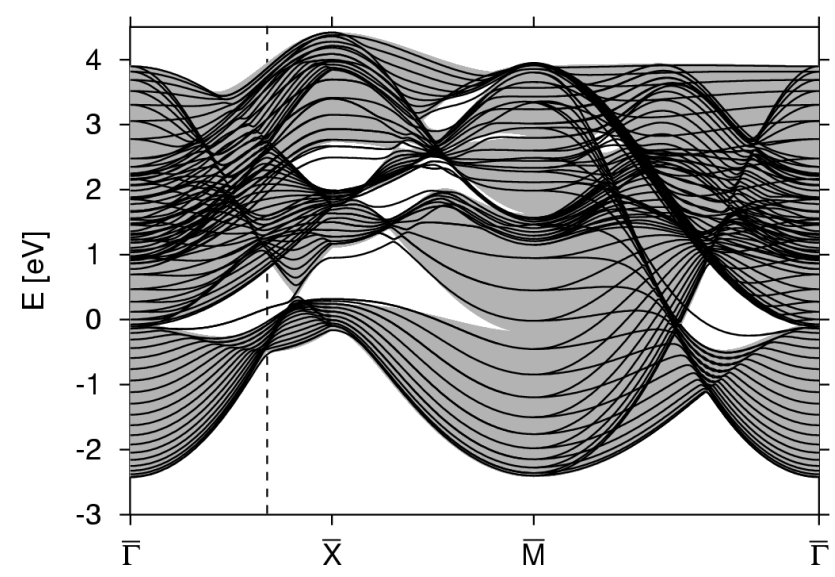

FIG. 2: Projected unoccupied (5d) $\uparrow$ band structure $(T=0 \mathrm{~K})$

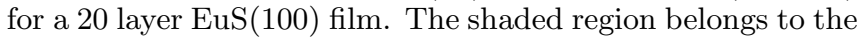
respective projected band structure of bulk EuS. The vertical broken line identifies the position in k-space of the surface state near the energy zero, which is investigated in Fig. 3. The energy zero has been chosen arbitrarily.

Fig. 2 shows the unoccupied (5d) $\uparrow$ band structure of a 20 layer $\operatorname{EuS}(100)$ film at $T=0 \mathrm{~K}$, calculated with the tight-binding-LMTO method. These results represent the single-particle input for our model calculation. Note that the energy zero has been chosen arbitrarily, not at all coinciding with the Fermi edge. For simplicity we restrict our presentation to the $5 \mathrm{~d}$ states only [1]. For comparison we also indicate in Fig. 2 the corresponding projected (5d) bulk-band structure, which has the density of states shown in the upper part of Fig. 2 in 11]. Above all that helps clearly to identify surface states. A surface state is a state which appears in the forbidden region where no bulk states occur. Another characteristic feature of a surface state is the exponential decay of its spectral weight with increasing distance from the surface. To demonstrate this, we have plotted in Fig. 3 the up-spin spectral density as function of energy for the cut, which is marked in Fig. 2 by a broken line, i. e. for the wave vector at $\frac{2}{3} \overline{\Gamma X}$. Furthermore, the spectral density is represented for different layers of the 20-layer film. $\alpha=1$ denotes the surface layer and $\alpha=10$ the middle layer. Note that the spectral density consists for $T=0$ and $\sigma=\uparrow$ exclusively of delta-functions representing quasiparticles of infinite lifetimes. Only to visualize the spectrum we have added a small imaginary part to the self energy getting therewith spectral density peaks of finite widths. The structure within the shaded region is obviously a surface state. Its spectral weight decreases exponentially with increasing distance of the respective layer from the surface. Another surface state appears at the bottom of the spectrum. We interpret this surface state as the analogue to that of $\mathrm{EuO}$ which has been speculated in ref. 9] to give rise to a surface metal-insulator transition when cooling down from $T_{\mathrm{C}}$ to $T=0$. Fig. 4 


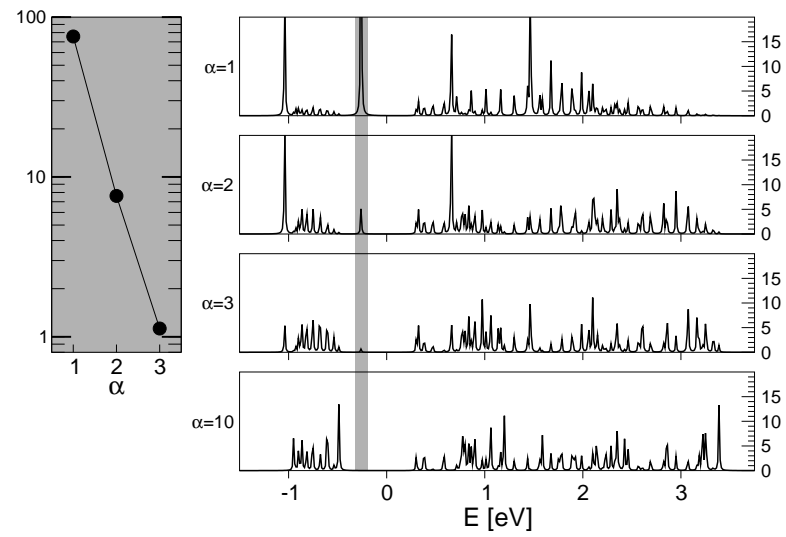

FIG. 3: Spectral density $(T=0, \sigma=\uparrow)$ as function of energy for the wave vector at $\frac{2}{3} \overline{\Gamma X}$ (dashed line in Fig. 2) and for different layers $(\alpha=1,2,3,10)$ of a 20-layer $\operatorname{EuS}(100)$ film. The inset shows on a logarithmic scale the spectral weight of the state within the grey column for the surface and the two layers next to the surface. $\alpha=1(10)$ means surface (middle) layer.

shows the temperature dependence of the spectral density, again for the wave vector at $\frac{2}{3} \overline{\Gamma X}$ to include the above-discussed surface state. At $T=0$ (ferromagnetic saturation) the spiky structure of the $\uparrow$ spectral density refers to quasiparticles of infinite lifetimes. The $\uparrow$ electron has no chance to exchange its spin with the parallel aligned localized $4 \mathrm{f}$ spins. It therefore propagates through the lattice without any scattering. On the other

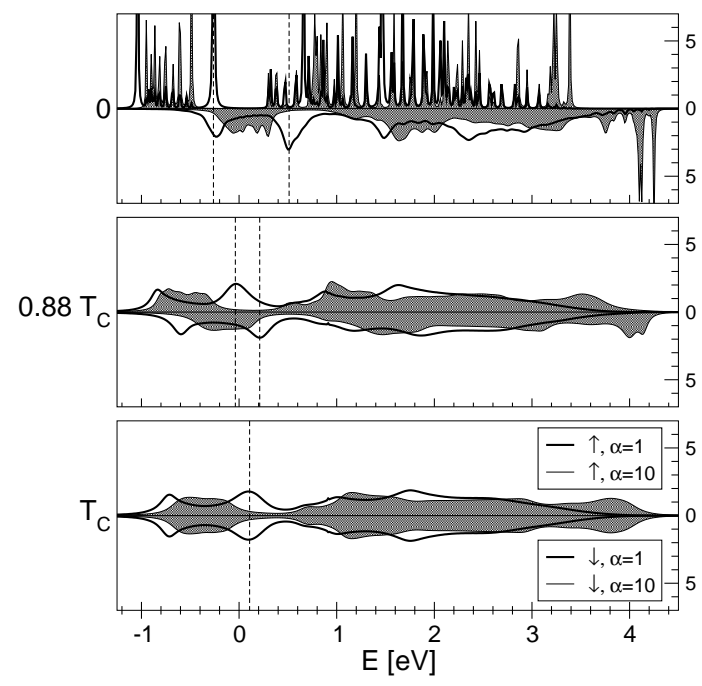

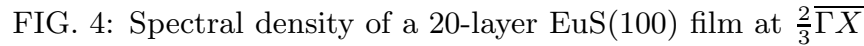
as function of energy and that for three different temperatures $\left(T=0,0.88 T_{\mathrm{C}}, T_{\mathrm{C}}\right)$. Upper halves for the up-spin spectrum, lower halves for the down-spin spectrum. Full lines: surface layer $(\alpha=1)$, thin lines (shaded regions): middle laver $(\alpha=$ 10). $T_{\mathrm{C}}=16.28 \mathrm{~K}$ for the 20 -layer film. The vertical broken lines indicate the position of the spin-split surface state; left line for $\sigma=\uparrow$, right line for $\sigma=\downarrow$. hand, a $\downarrow$ electron can be scattered by magnon emission or can form a magnetic polaron what gives rise to a dramatic lifetime broadening of the spectrum even at $T=0$. We want to stress that the $(T=0, \sigma=\downarrow)$ calculation is exact: For finite temperature, i. e. finite magnon densities, the $\uparrow$ electron, too, can be scattered by magnon absorption with a concomitant spin flip. Such processes lead to a rather structureless 5d excitation spectrum, which, nevertheless, carries a distinct layer dependence. The surface state, identified in Fig. 3. is spin split at $T=0 \mathrm{~K}$ by some $0.8 \mathrm{eV}$ due to the exchange coupling to the ferromagnetic $4 \mathrm{f}$ moment system. The induced exchange splitting is strongly temperature-dependent with a Stoner-like collapsing for $T \rightarrow T_{\mathrm{C}}$. The same behavior is shown up by the surface state at the bottom of the spectrum. An analogous feature has been reported for $\mathrm{EuO}$ in ref. 9]. This distinct temperature dependence in the ferromagnetic phase appears at first glance somewhat astonishing because it happens to the unoccupied and "a priori" uncorrelated $5 d$ energy states of semiconducting EuS.

By a combination of an LDA-band structure calculation with a many-body evaluation of the multiband Kondo-lattice model a pronounced temperature dependence of the (empty) $5 d$ conduction bands of $\operatorname{EuS}(100)$ films could be demonstrated. Exchange split magnetic surface states show up a Stoner-like collapsing for $T \rightarrow$ $T_{\mathrm{C}}$. The Curie temperature exhibits a characteristic filmthickness dependence understandable as a typical finite size effect.

Financial support by the SFB 290 of the "Deutsche Forschungsgemeinschaft" is gratefully acknowledged.

* URL: http://tfk.physik.hu-berlin.de

[1] P. Wachter, Handbook of the Physics and Chemistry of Rare Earth (Amsterdam, North Holland, 1979), vol. 1, chap. 19.

[2] H. G. Bohn, W. Zinn, B. Dorner, and A. Kollmar, Phys. Rev. B 22, 5447 (1980).

[3] A. Stachow-Wójcik, T. Story, W. Dobrowolski, M. Arciszewska, R. R. Gałązka, M. W. Kreijveld, C. H. W. Swüste, H. J. M. Swagten, W. J. M. de Jonge, A. T. Twardowski, et al., Phys. Rev. B 60, 15220 (1999).

[4] T. W. Capehart and M. E. Fisher, Phys. Rev. B 13, 1133 (1976).

[5] M. Farle, K. Baberschke, U. Stetter, A. Aspelmeier, and F. Gerhardter, Phys. Rev. B 47, 11571 (1993).

[6] D. Weller, S. F. Alvarado, W. Gudat, K. Schröder, and M. Campagna, Phys. Rev. Lett. 54, 1555 (1985).

[7] P. G. Steeneken, L. H. Tjeng, I. Elfimov, G. A. Sawatzky, G. Ghiringhelli, N. B. Brookes, and D. J. Huang, Phys. Rev. Lett. 88, 047201 (2002).

[8] R. Schiller, W. Müller, and W. Nolting, Phys. Rev. B 64, 134409 (2001).

[9] R. Schiller and W. Nolting, Phys. Rev. Lett. 86, 3847 (2001). 
[10] N. M. Mermin and H. Wagner, Phys. Rev. Lett. 17, 1133 (1966).

[11] W. Müller and W. Nolting, Phys. Rev. B 66, 085205 (2002).

[12] R. Schiller and W. Nolting, Solid State Commun. 118, 173 (2001).

[13] O. K. Andersen, Phys. Rev. B 12, 3060 (1975).

[14] O. K. Andersen and O. Jepsen, Phys. Rev. Lett. 53, 2571 (1984).
[15] R. Schiller, W. Müller, and W. Nolting, J. Magn. Magn. Mater. 169, 39 (1997).

[16] W. Nolting, S. Rex, and S. Mathi Jaya, J. Phys.: Condens. Matter 9, 1301 (1997).

[17] W. Nolting, G. G. Reddy, A. Ramakanth, D. Meyer, and J. Kienert, Phys. Rev. B 67, 024426 (2003).

[18] T. Hickel and W. Nolting, Phys. Rev. B submitted (2003). 